\title{
Comparison of AIMS65 and Glasgow Blatchford scores in predicting mortality in patients with upper gastrointestinal bleeding
}

\author{
Rohat $A k^{1 *}$ (1), Nihat Müjdat Hökenek² (1)
}

\section{SUMMARY}

OBJECTIVE: Several mortality prediction scores are available for patients with upper gastrointestinal bleeding who visited the emergency department; however, most of the available scores include endoscopic data. Endoscopy is difficult or impossible to access for many emergencies departments worldwide. The aim of this study was to evaluate and compare the performance of the albumin, INR, alteration in mental status, systolic blood pressure and age 65 score and the Glasgow-Blatchford score in predicting mortality in patients with upper gastrointestinal bleeding who visited the emergency department and for which endoscopic data were not required.

METHODS: The data of patients with acute upper gastrointestinal bleeding who visited the emergency department during the study period were retrospectively analyzed. The data were obtained from the hospital automation system using the international classification of disease codes via computer registration. The prediction accuracy of AIMS65 and Glasgow-Blatchford score was compared using the area under the receiver operating characteristic curve method.

RESULTS: There were 422 patients in total; the mean age of these patients was 68.5 while $62.6 \%$ were males. The mortality rate was 30 (7.1\%). The AIMS65 score performed better with an AUC 0.706 [95\% CI 0.660-0.749; $p<0.001]$ compared with the Glasgow-Blatchford score (AUC 0.542; 95\% CI 0.4693-0.576; $p=0.11$ ).

CONCLUSION: In this study, it was revealed that AIMS65, which is a score that can be easily calculated only with the data in the emergency department, outperformed Glasgow-Blatchford score in predicting mortality in patients with acute upper gastrointestinal bleeding who visited the emergency department.

KEYWORDS: Assessment, risk. Emergency care. Gastrointestinal endoscopy. Mortality. Upper gastrointestinal tract.

\section{INTRODUCTION}

Acute upper gastrointestinal bleeding (UGIB) is a common cause of hospitalization and a disease with high mortality rates in the emergency department (ED). Despite the advances in intensive care technologies and the endoscopic treatment of UGIB, mortality remains a major problem. It has been reported that the overall rate of mortality due to the disease ranges from $3-15 \%$. These rates increase even further for those in an unstable hemodynamic condition ${ }^{1-3}$.

The guidelines from the American College of Gastroenterology recommend the use of early risk scores in the management of patients with acute $\mathrm{UGIB}^{4}$. The number of published risk scoring systems for these patients has increased rapidly in recent years ${ }^{5-7}$. Among these, the AIMS65 score, developed by Saltzman et al. ${ }^{8}$, includes five variables, namely, albumin, international normalized ratio (INR), altered mental status, systolic blood pressure, and an age above 65 years. The values of the scores can range from $0-5$ and are used to estimate in-hospital mortality $^{8}$. The Glasgow-Blatchford score (GBS) includes eight variables, namely, blood urea nitrogen (BUN), hemoglobin level, systolic blood pressure, pulse, melena, syncope, liver disease, and heart failure. Any score higher than zero in this score is

\footnotetext{
IIstanbul Kartal Dr. Lutfi Kirdar City Hospital, Department of Emergency Medicine - Istanbul, Turkey.

${ }^{2}$ Kartal Dr. Lütfi Kırdar City Hospital, Department of Emergency Medicine - Istanbul, Turkey.

*Corresponding author: rohatakmd@gmail.com

Conflicts of interest: the authors declare there are no conflicts of interest. Funding: none.

Received on June 11, 2021. Accepted on June 27, 2021.
} 
interpreted as the risk of needing a transfusion, endoscopy, or surgical intervention?.

This study aims to evaluate and compare the performances of the AIMS65 and GBS in predicting in-hospital mortality in patients with UGIB who visited the ED.

\section{METHODS}

This retrospective observational study was carried out in the ED of a tertiary care teaching hospital between January 1, 2019 - January 1, 2020. The electronic patient database of the hospital was scanned for the determined period of this study; patients above the age of 18 , who were diagnosed with UGIB and hospitalized after visiting the ED according to the codes of the International Classification of Diseases (ICD) 10th revision, were included in this study. The definition of acute UGIB was based on the presence of at least one of the following three features: hematemesis, melena, or solid clinical evidence and laboratory support for acute blood loss from the upper gastrointestinal (UGI) tract ${ }^{10}$. Patients with missing records, patients transferred from other hospitals, patients with variceal bleeding, patients with records of less than 30 days, and patients with a diagnosis other than UGIB after hospitalization were excluded from this study. The institutional review board approved the analysis and issued a waiver of consent (Ethics Committee Ruling number: 2021/514/200/16).

The following data were collected for each patient: age, gender, information about their mental status, symptoms at admission (i.e., hematemesis, coffee-like substance vomiting, melena, syncope, lethargy, blood pressure, and pulse), comorbidities (i.e., ischemic heart disease, diabetes mellitus, congestive heart failure, and liver disease), medications, and laboratory results (i.e., albumin levels, INR, BUN, and hemoglobin). If the Glasgow Coma Scale score of the patients was $<14$ or their consciousness status included disorientation (i.e., lethargy, stupor, or coma), then their mental status was considered to have changed. AIMS65 and GBS scores were calculated for each patient as previously defined ${ }^{8,9}$. The data for each scoring system were entered into an Excel database (Microsoft Inc., Richmond, WA, USA) and analyzed by one of the researchers. After the data analysis, the other researcher presented quality improvement feedback. The primary outcome of this study was 30-day all-cause in-hospital mortality.

\section{Statistical analysis}

All calculations were performed using SPSS 15.0 for Windows and MedCalc. Descriptive criteria include the median and interquartile range values and are presented as a percentage distribution. The conformity of the data to the normal distribution was verified with the Kolmogorov-Smirnov test. Receiveroperating characteristic (ROC) curves for 30-day mortality were calculated for AIMS65 and GBS, and the predictive accuracy of each scoring system was measured by the area under the receiver-operating curve (AUC). The method established by DeLong et al. was used to compare the ROC curves of the risk scores ${ }^{11}$. This study was completed with a $95 \% \mathrm{CI}$ and statistical significance was accepted as $\mathrm{p}<0.05$.

\section{RESULTS}

Among the 558 patients admitted with the definition of acute UGIB during the study period, 136 individuals were excluded based on the exclusion criteria. The data from the total of 422 patients with acute UGIB were then recorded, retrospectively. In this study, $62.6 \%$ of the patients are men while the median age is 68.5 years. More than half of the patients $(74.2 \%)$ had melena as an admission symptom (Table 1 ).

Mortality occurred in 30 (7.1\%) of the 422 patients who were included in this study (Table 1). While death occurred in $2.7 \%$ of patients with an AIMS65 score value of 0 , the mortality rate for values of $1,2,3,4$, and 5 is $6.2,8.5,46.2,50.0$, and $100 \%$, respectively (Table 2 ). As a result of the ROC analysis, the AUC was determined to be 0.706 (95\%CI 0.660-0.749) for the AIMS65 score. The sensitivity of predicting the probability of death in individuals with a value above 0 was $50.0 \%$ and the selectivity was $78.8 \%$; it was found to be statistically significant $(\mathrm{p}=0.001)$. In the GBS, patients with values between $0-5$ had a mortality rate of $9.1 \%$, while the values between $6-12$ and $13-23$ had mortality rates of 5.3 and $9.7 \%$, respectively. As a result of the ROC analysis, the AUC was found to be $0.542(95 \% \mathrm{CI}$ $0.4693-0.576$ ), and the value of GBS in predicting mortality was not found to be statistically significant $(\mathrm{p}=0.11)$ (Figure 1 ).

\section{DISCUSSION}

UGIB is a common gastrointestinal emergency that can cause high morbidity and mortality at a rate of $3-10 \%{ }^{12,13}$. There are more than 300,000 hospital admissions per year in the USA due to $\mathrm{UGIB}^{14}$. In the United Kingdom, the rate is $103-172 / 100,000$ admissions per year, with a mortality rate of $8-14 \%{ }^{15}$. It is important to determine the necessary level of care such as early endoscopy, early surgical/interventional radiological procedures, or intensive care for a medical emergency with such a high mortality rate. Many international guidelines suggest early risk stratification to determine the appropriate care of patients who are admitted to the ED with $\mathrm{UGIB}^{16}$. The results of this study demonstrate that the AIMS65 score is superior to the GBS in the context of 30-day in-hospital mortality in patients with UGIB. 
Medical treatment in EDs must be managed rapidly. Some patients identified as being at high risk of death may be given priority for a transfusion and hospital admission after UGIB.

Table 1. Characteristics of patients.

\begin{tabular}{|c|c|}
\hline Baseline characteristics & $\begin{array}{c}\text { Median (IQR) or } n \\
(\%)\end{array}$ \\
\hline Age & $68.5(27)$ \\
\hline Male, n (\%) & $264(62.6)$ \\
\hline \multicolumn{2}{|l|}{ Clinical parameters } \\
\hline Heart rate (beats/min) & $98(15)$ \\
\hline $\mathrm{SBP}(\mathrm{mmHg})$ & $120(20)$ \\
\hline $\mathrm{DBP}(\mathrm{mmHg})$ & $65(12)$ \\
\hline \multicolumn{2}{|l|}{ Laboratory results } \\
\hline $\mathrm{Hb}(\mathrm{mg} / \mathrm{dL})$ & $8.8(2.5)$ \\
\hline Platelet count (dL) & $212.5(114)$ \\
\hline $\mathrm{BUN}(\mathrm{mg} / \mathrm{dL})$ & $63(55)$ \\
\hline $\mathrm{Cr}(\mathrm{mg} / \mathrm{dL})$ & $0.8(0.4)$ \\
\hline Albumin (mg/dL) & $29(15)$ \\
\hline INR & $1.2(0.3)$ \\
\hline \multicolumn{2}{|l|}{ Presenting symptoms } \\
\hline Hematemesis, n (\%) & $84(19.9)$ \\
\hline Melena, n (\%) & $313(74.2)$ \\
\hline Hematochezia, n (\%) & $58(13.7)$ \\
\hline Mental status change, n (\%) & $7(1.7)$ \\
\hline Syncope, n (\%) & $24(5.7)$ \\
\hline \multicolumn{2}{|l|}{ Comorbid illness } \\
\hline Liver disease, n (\%) & $9(2.1)$ \\
\hline Congestive heart failure, $\mathrm{n}(\%)$ & $38(9)$ \\
\hline Renal disease, n (\%) & $24(5.7)$ \\
\hline Diabetes mellitus, n (\%) & $90(21.3)$ \\
\hline Hypertension, n (\%) & $170(40.3)$ \\
\hline \multicolumn{2}{|l|}{ Medications } \\
\hline Clopidogrel, n (\%) & $26(6.2)$ \\
\hline Warfarin, n (\%) & $40(9.5)$ \\
\hline $\begin{array}{l}\text { Nonsteroidal anti- } \\
\text { inflammatory drugs, } \mathrm{n}(\%)\end{array}$ & $83(19.7)$ \\
\hline Acetylsalicylic acid, n (\%) & $97(23)$ \\
\hline Length of hospital stay (d) & 3 \\
\hline Mortality, n (\%) & $30(7.1)$ \\
\hline
\end{tabular}

SBP: systolic blood pressure; DBP: diastolic blood pressure; Hb: hemoglobin; ALB: albumin; BUN: blood urine nitrogen; Cr: creatinine; INR: international normalized ratio.
Many scoring systems have been developed that can be used for this purpose. However, most of the current scoring systems are not useful for EDs since they are based on endoscopic data. Moreover, many EDs worldwide do not have access to endoscopy.

Risk predicting models to be used in the ED should be easily calculated and reproducible. The AIMS65 score is a

Table 2. Distribution of patients and 30-day mortality in each scoring systems.

\begin{tabular}{l|c|c}
\multicolumn{1}{|c|}{$\begin{array}{c}\text { Number } \\
\text { of patients } \\
(\mathrm{n}=422)\end{array}$} & $\begin{array}{c}30 \text { day mortality } \\
(\mathrm{n}=30)\end{array}$ \\
\hline AlMS65 score & $147(34.8)$ & $4(2.7)$ \\
\hline 0 & $177(41.9)$ & $11(6.2)$ \\
\hline 1 & $82(19.4)$ & $7(8.5)$ \\
\hline 2 & $13(3.1)$ & $6(46.2)$ \\
\hline 3 & $2(0.5)$ & $1(50)$ \\
\hline 4 & $1(0.2)$ & $1(100)$ \\
\hline 5 & & 0.001 \\
\hline$p-v a l u e$ & $22(5.2)$ & $2(9.1)$ \\
\hline Glasgow-Blatchford score & $13(5.3)$ \\
\hline $0-5$ & $246(58.3)$ & $15(9.7)$ \\
\hline $6-12$ & $154(36.5)$ & 0.23 \\
\hline $13-23$ & &
\end{tabular}

p-value: $\chi^{2}$ test for 30 -day mortality.

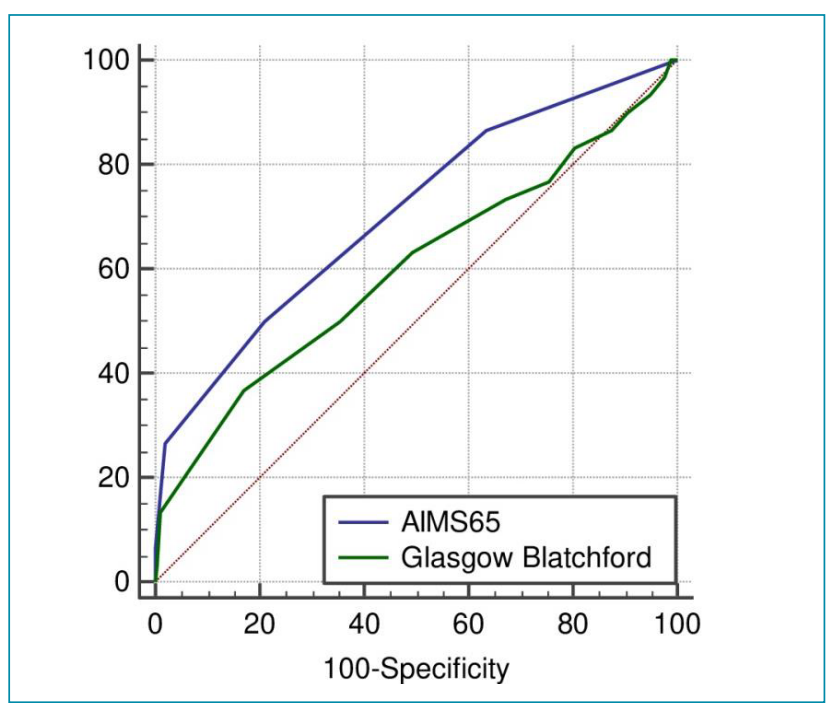

Figure 1. Receiver-operating characteristic curves comparing the prediction of 30-day in-hospital mortality in patients with acute upper gastrointestinal bleeding based on the AIMS65 and Glasgow-Blatchford scores. 
prognostic scoring system with good performance, especially in the early diagnosis of patients with UGIB who have a high risk of in-hospital mortality ${ }^{8}$. The AIMS65 score is a five-variable prediction tool using physical examination findings, vital signs, routine laboratory values, and age; it provides risk prediction in EDs without emergency endoscopy. Similarly, GBS is based on simple clinical and laboratory parameters and does not require emergency endoscopy 9 . However, the GBS is not useful for routine clinical practice due to its limitations: some scoring items from the medical history of a patient may not readily be available in EDs.

In this study, AIMS65 performed well in predicting mortality with an AUC 0.706 . GBS was found to be ineffective in predicting mortality (AUC 0.542). In a study by Stanley et al., the AIMS65 score was found to be superior to GBS (AUC 0.69) with an AUC $0.78^{5}$. A study conducted by Min Park et al., which had a sample size of 523 patients, reported that the AIMS65 (AUC 0.79) was superior to GBS (AUC 0.61) ${ }^{17}$. Additionally, in the study conducted by Yaka et al., with 254 patients, it was emphasized that the AIMS65 (AUC 0.849) was more successful than GBS (AUC 0.809) in predicting mortality ${ }^{18}$.

We asserted that the disappointing performance of GBS in predicting mortality can be explained by the fact that this risk scoring system was originally developed to predict the requirement for intervention (i.e., transfusion, endoscopy, or surgery) and not for mortality prediction. There are some limitations to this study. First, this is a single-center study conducted on a relatively small population and requires confirmation in a larger, multi-center cohort. In addition, due to the retrospective nature of this study, the data were obtained from an electronic registry system; this limited the amount of data able to be implemented due to incomplete or outdated information.

\section{CONCLUSIONS}

In this study, it was found that the AIMS65 score, which can be easily calculated with only the data from the ED, outperformed GBS in predicting mortality in patients with acute UGIB admitted to the ED. However, more multi-center and prospective studies are required to demonstrate the wider applicability of this score.

\section{AUTHORS' CONTRIBUTIONS}

RA: Conceptualization, Data curation, Formal analysis, Funding acquisition, Investigation, Methodology, Project administration, Resources, Software, Supervision, Writing - original draft. NMH: Validation, Visualization, Writing -review \& editing.

\section{REFERENCES}

1. Tang $Y$, Shen J, Zhang F, Zhou $X$, Tang Z, You T. Scoring systems used to predict mortality in patients with acute upper gastrointestinal bleeding in the ED. Am J Emerg Med. 2018;36(1):27-32. https://doi.org/10.1016/j.ajem.2017.06.053

2. Li Y, Song Y. Diagnostic value of serum gastrin and epidermal growth factor to the gastric ulcer complicated with upper gastrointestinal hemorrhage. J Coll Physicians Surg Pak. 2020;30(12):1269-72. https://doi.org/10.29271/ jcpsp.2020.12.1269

3. Laine L, Yang H, Chang SC, Datto C. Trends for incidence of hospitalization and death due to $\mathrm{Gl}$ complications in the United States from 2001 to 2009. Am J Gastroenterol. 2012;107(8):1190-5;quiz1196. https://doi.org/10.1038/ ajg.2012.168

4. Laine $L$, Jensen DM. Management of patients with ulcer bleeding. Am J Gastroenterol. 2012;107(3):345-60;quiz361. https://doi.org/10.1038/ajg.2011.480

5. Stanley AJ, Laine L, Dalton HR, Ngu JH, Schultz M, Abazi $\mathrm{R}$, et al. Comparison of risk scoring systems for patients presenting with upper gastrointestinal bleeding: international multicentre prospective study. BMJ. 2017;356:i6432. https:// doi.org/10.1136/bmj.i6432

6. Oakland K, Jairath V, Uberoi R, Guy R, Ayaru L, Mortensen $N$, et al. Derivation and validation of a novel risk score for safe discharge after acute lower gastrointestinal bleeding: a modelling study. Lancet Gastroenterol Hepatol. 2017;2(9):63543. https://doi.org/10.1016/S2468-1253(17)30150-4

7. Gu L, Xu F, Yuan J. Comparison of AIMS65, Glasgow-Blatchford and Rockall scoring approaches in predicting the risk of in-hospital death among emergency hospitalized patients with upper gastrointestinal bleeding: a retrospective observational study in Nanjing, China. BMC Gastroenterol. 2018;18(1):98. https://doi.org/10.1186/s12876-018-0828-5

8. Saltzman JR, Tabak YP, Hyett BH, Sun X, Travis AC, Johannes RS A simple risk score accurately predicts in-hospital mortality, length of stay, and cost in acute upper $\mathrm{Gl}$ bleeding. Gastrointest Endosc. 2011;74(6):1215-24. https://doi.org/10.1016/j.gie.2011.06.024

9. Blatchford O, Murray WR, Blatchford M. A risk score to predict need for treatment for upper-gastrointestinal haemorrhage. Lancet. 2000;356(9238):1318-21. https://doi.org/10.1016/ S0140-6736(00)02816-6

10. Hearnshaw SA, Logan RF, Lowe D, Travis SP, Murphy MF, Palmer KR. Acute upper gastrointestinal bleeding in the UK: patient characteristics, diagnoses and outcomes in the 2007 UK audit. Gut. 2011;60(10):1327-35. https://doi.org/10.1136/ gut.2010.228437

11. DeLong ER, DeLong DM, Clarke-Pearson DL. Comparing the areas under two or more correlated receiver operating characteristic curves: a nonparametric approach. Biometrics. 1988;44(3):837-45. https://doi.org/10.2307/2531595 
12. Button LA, Roberts SE, Evans PA, Goldacre MJ, Akbari $A$, Dsilva $R$, et al. Hospitalized incidence and case fatality for upper gastrointestinal bleeding from 1999 to 2007: a record linkage study. Aliment Pharmacol Ther. 2011;33(1):64-76. https://doi.org/10.1111/j.13652036.2010.04495.x

13. Gralnek IM, Barkun AN, Bardou M. Management of acute bleeding from a peptic ulcer. N Engl J Med. 2008;359(9):92837. https://doi.org/10.1056/NEJMra0706113

14. Kurien M, Lobo AJ. Acute upper gastrointestinal bleeding. Clin Med (Lond). 2015;15(5):481-5. https://doi.org/10.7861/ clinmedicine.15-5-481

15. Rockall TA, Logan RF, Devlin HB, Northfield TC. Incidence of and mortality from acute upper gastrointestinal haemorrhage in the United Kingdom. Steering Committee and members of the National Audit of Acute Upper Gastrointestinal
Haemorrhage. BMJ. 1995;311(6999):222-6. https://doi. org/10.1136/bmj.311.6999.222

16. Sung JJ, Chiu PW, Chan FKL, Lau JY, Goh KL, Ho LH, et al. Asia-Pacific working group consensus on non-variceal upper gastrointestinal bleeding: an update. Gut. 2018;67(10):175768. https://doi.org/10.1136/gutjnl-2018-316276

17. Park SM, Yeum SC, Kim BW, Kim JS, Kim JH, Sim EH, et al. Comparison of AIMS65 score and other scoring systems for predicting clinical outcomes in Koreans with nonvariceal upper gastrointestinal bleeding. Gut Liver. 2016;10(4):52631. https://doi.org/10.5009/gnl15153

18. Yaka E, Yılmaz S, Doğan NÖ, Pekdemir M. Comparison of the Glasgow-Blatchford and AIMS65 scoring systems for risk stratification in upper gastrointestinal bleeding in the emergency department. Acad Emerg Med. 2015;22(1):22-30. https://doi.org/10.1111/acem.12554 